\title{
Fast Single Image Haze Removal Method based on Atmospheric Scattering Model
}

\author{
De-Wei Huang, Ke-Xin Chen \\ College of Electronic Engineering, South China \\ Agricultural University, China \\ E-mail: huangdewei@stu.scau.edu.cn, \\ 285078375@qq.com
}

\author{
Jian-Qiang Lu, Wei-Xing Wang \\ College of Electronic Engineering, South China \\ Agricultural University, China \\ Guangdong Engineering Research Center for Monitoring \\ Agricultural Information, China \\ Key Laboratory of Information Acquisition and \\ Application in Agriculture, Guangzhou Science \\ Technology and Innovation Commission, China \\ E-mail: Ljq@scau.edu.cn,weixing@scau.edu.cn
}

\begin{abstract}
In this paper, we propose an effective and efficient method to remove haze from a single input image. We first remove fine details from the minimum channel of RGB channels by a low-pass filter and then use it as a rough estimation of the transmission map. Then, we refine the rough estimation by using the saturation channel of HSI color space and local contrast. Based on the atmospheric scattering model, we then obtain a high-quality haze-removed image. Results on a variety of hazy images demonstrate the effectiveness and efficiency of our method.
\end{abstract}

Keywords-dehaze; saturation; low-pass filter; contrast; transmission map estimation

\section{INTRODUCTION}

With the development of computer technology, haze removal is getting more and more attention in consumer/computational photography and computer vision applications (like remote sense, driving assistance and video surveillance). Under bad weather conditions, images of outdoor scenes are usually degraded by the particles (like haze, fog and smoke) suspended in the atmosphere. Due to the scattering effect of suspended particles to lights, the reflected lights received by the camera from the scene point are attenuated while being blended with air-lights -ambient lights scattered by atmospheric particles. Eventually, images are captured with decreased visibility and color shift. Removing haze can significantly increase the visibility and correct the color shift mentioned above. However, haze removal is an ill-posed problem for lacking information about haze-free images.

Nowadays, dehazing methods can be divided into two categories. One type is mainly based on image enhancement which can obtain good visibility but tend to cause large distortion. The other type is mainly based on the atmospheric scattering model [1] which analyzed the mechanism of image degradation by haze. This type of method can obtain haze-removed images that are closer to the 'real' haze-free images. The one based on the atmospheric scattering model is commonly used. Some removed haze by getting information about scene depth from different ways, like user interaction [2], known 3D model [3] or multi-images under different atmospheric conditions [4-6]. Some others removed haze by using multi-images took under different polarization angles [7-8]. However, all the method in [4-8] can hardly be used in applications due to the harsh requirements. Recently, dehazing methods using only single image are developed. The success of these methods lies on strong priors or assumptions. Fattal [9] removed haze under the assumption of local uncorrelation between the medium transmission and the albedo of the scene. When dealing with thin fog images, this method performs well. However, this method can't handle dense fog images well. Moreover, the local uncorrelation assumption is not always true. Tarel [10] analyzed the range of atmospheric veil and assumed atmospheric veil changing slowly to remove haze. This method can significantly remove haze while causing residual haze, halo effect and chromatic aberration. He [11] proposed a dark channel prior to roughly estimate the transmission followed by a soft matting process to optimize the transmission. This method performs well while being very time-consuming and not handling well images with large area of sky. Later, He [12] proposed a guided filter to replace the soft-matting which greatly reduced the processing time but still not fast enough and slightly weakened the effectiveness. Wang [13] used a method of multiscale transmission-map fusion, which performing well but being extreme time-consuming. Zhu [14] proposed a color attenuation prior and formed a linear equation about the minimum channel of RGB channels and scene depth which was obtained through a training with synthesis hazy images. This method can remove haze very fast but can only handle thin haze images well and can't achieve vivid details.

In this paper, we propose a new method to remove haze from a single input image to have a good performance both on the effectiveness and efficiency. We first remove fine details from the minimum channel of RGB channels by a low-pass filter. Then we use it as a rough estimation of the transmission map followed by a refinement using the saturation channel of HSI color space and local contrast. Based on the atmospheric scattering model, we then obtain a high-quality haze-removed image. At the end, experiments on different images are implemented to compare with others' methods with quantitation measurements. 


\section{OUR METHOD}

In this section, we first introduce the widely used atmospheric scattering model, then present our method. At the end, we conclude the procedures of our method.

\section{A. Atmospheric Scattering Model}

This model can be expressed as

$$
\begin{gathered}
I^{c}(x)=J^{c}(x) t(x)+A(1-t(x)) \\
t(x)=e^{-\beta d(x)}
\end{gathered}
$$

where $C \in\{R, G, B\}$ for RGB images, I is a hazy image, $\mathrm{J}$ is the haze-free image of $\mathrm{I}, \mathrm{t}$ is the medium transmission, A is the global atmospheric light, $\beta$ is the scattering coefficient of the atmosphere, and $d$ is the scene depth. Equation (1) shows that the unknown variables $t$ and A have to be estimated in order to recover $\mathrm{J}$ from $\mathrm{I}$.

\section{B. Medium Transmission Estimation}

Normally, most pixels in hazy images have a lower value than $\mathrm{A}$. According to the dark channel prior of $\mathrm{He}$ [15], at least one channel of an out-door color (RGB) image has a low value for most pixels, which is normally the case due to shadows, colorful objects and dark objects. It is obvious to see from equation (1) that the minimum value of RGB channels of I, which is denoted as W0 in this paper, increases with decreasing $t$. This means that we can get a rough estimation about $t$ via W0. He [11] first used a minimum filter to $\mathrm{W} 0$ to get a rough estimation about $t$, then refined the rough estimation by a guided filter. Tarel [10] first used W0 directly as the rough estimation of $t$, then refined it by two median filters. Zhu [14] estimated t using a linear equation about W0 and scene depth d obtained through a training with synthesis hazy images.

Unlike Tarel [10], we don't directly use W0 as a rough estimation of $\mathrm{t}$ since $\mathrm{W} 0$ contains much fine details which are not good for the estimation of $t$ since $t$ should vary smoothly except for areas with depth discontinuity. We extract the fine details and then subtract it from W0.

$$
\begin{gathered}
V(x)=A^{*}(1-t)=W(x) \\
W(x)=W_{0}(x)-H \otimes W_{0}(x) \\
W_{0}(x)=\min _{c \in\{R, G, B\}} I^{C}(x) \\
H=\frac{1}{8}\left[\begin{array}{ccc}
-1 & -1 & -1 \\
-1 & 8 & -1 \\
-1 & -1 & -1
\end{array}\right]
\end{gathered}
$$

Where $\otimes$ denotes a convolution operation. Then, the refinement of $t$ needs to be done. By analyzing equation (1) again, we find that the pixel value $\mathrm{I}^{\mathrm{C}}(\mathrm{x})$ gets closer to $\mathrm{A}$ when the value of $t$ gets smaller, which means that the disparity between RGB color channels decreases with $t$ and the pixel is more affected by haze. By converting RGB color space to HSI color space, we find that the saturation $\mathrm{S}$ of a pixel is smaller when it is more affected by haze. Consequently, smaller the saturation $\mathrm{S}$ of a pixel is, the more reliable using $\mathrm{W}$ as the estimation of $\mathrm{t}$ is. We model the credibility function of $\mathrm{S}$ as a Gaussian function with mean value of 0 ,

$$
\begin{aligned}
& P_{x}(S)=e^{-\frac{S^{2}}{2 \sigma^{2}}} \\
& S=1-\frac{W(x)}{\operatorname{Int}(x)}
\end{aligned}
$$

$$
\operatorname{Int}(x)=\frac{\sum_{C \in\{R, G, B\}} I^{C}(x)}{3}
$$

where $\sigma$ is set to be 0.5 for all the experiments in this paper. Hence, for the estimation of $\mathrm{t}$, we have the following equation,

$$
V(x)=P_{x}(s) * W(x)
$$

However, using this equation to estimate $t$ is not nough considering that the white objects in input images may be distorted to gray objects due to small estimated value of $t$ for them. So we need to refine equation (8) by ultiplying a term $\mathrm{C}(\mathrm{x})$ for preserving the white objects. By ervation, we find that white objects normally are much smoother locally than haze, which means that the local contrast of white objects is much lower than that of haze. $\mathrm{C}(\mathrm{x})$ will set the estimated value of $t$ for white objects to be lower since it has a lower contrast. The three normally used quantitative definitions of contrast are Mean Squared Error (MSE) Contrast, Michelson Contrast and Weber Contrast.

$$
\begin{gathered}
C_{\text {MSE }}=\sum_{y \in P(x)}^{N} \frac{(W(y)-\overline{W(x)})^{2}}{N} \\
C_{\text {Meichelson }}=\frac{W(x)_{\max }-W(x)_{\min }}{W(x)_{\max }+W(x)_{\min }} \\
C_{\text {Weber }}=\sum_{y \in P(x)}^{N} \frac{W(y)-\overline{W(x)}}{N^{*} W(x)}
\end{gathered}
$$

Where $\mathrm{P}(\mathrm{x})$ is a local patch centered at pixel $\mathrm{x}, \mathrm{N}$ is the 
total number of pixels in the patch $\mathrm{P}(\mathrm{x}), \overline{\mathrm{W}(\mathrm{x})}$ is the mean value among the patch $P(x)$. The patch size is set to be $5 \times 5$ and the definition of contrast we use is the MSE contrast for all the experiments in this paper. Normally, the $\mathrm{C}_{\mathrm{MSE}}$ is much small than 1 . Hence, we normalize it to enhance the difference between $\mathrm{C}_{\mathrm{MSE}}$ by simply dividing the maximum of $\mathrm{C}_{\mathrm{MSE}}$,

$$
C_{M S E, N}=\frac{C_{M S E}}{\max \left\{C_{M S E}\right\}}
$$

Similarly, we use a Gaussian function with a mean value of 1 to express $\mathrm{C}(\mathrm{x})$,

$$
C(x)=e^{-\frac{\left(C_{M S E, N}-1\right)^{2}}{2 \sigma^{2}}}
$$

Where $\sigma$ is set to be $\sqrt{5}$ which leading $\frac{1}{2 \sigma^{2}}$ to be 0.1 and then the minimum of $\mathrm{C}(\mathrm{x})$ is about 0.9 which can't be too small in order to ensure a sufficient degree of haze removing. Finally, we have the following equation,

$$
V(x)=C(x) * P_{x}(s) * W(x)
$$

For explicitly expressing t, we have

$$
t(x)=1-\frac{V(x)}{A}
$$

Then $\mathrm{J}(\mathrm{x})$ can be expressed as
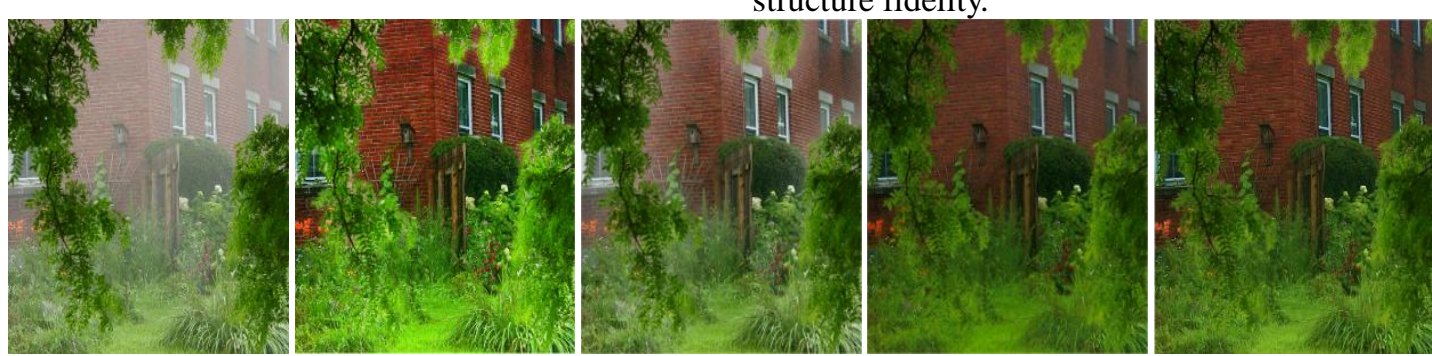

Figure 1. From left to right: input hazy image, restored image by Tarel, He, Zhu, our method.
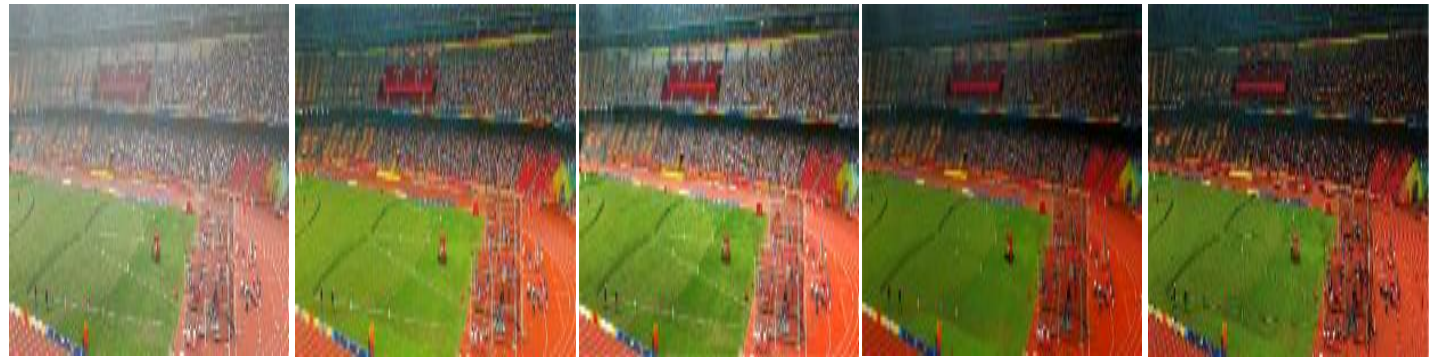

Figure 2. From left to right: input hazy image, restored image by Tarel, He, Zhu, our method. amplifies the noise. After estimating A, we can then recover $\mathrm{J}$ according to (18).

\section{Global Atmospheric Light Estimation}

According to Nayar [1], the best estimate of A is the top $0.1 \%$ brightest pixels in the dark channel among which imple way to overcome this problem by averaging the $0.1 \%$ in sky and white objects. Like this method, we use than RGB channels.

\section{Procedures of our method:}

1: get A by using the intensity channel;

channel;

3: get $\mathrm{W}$ according to equation (4) (6);

4: get $t$ according to equation (7), (10), (11), (14) (17);

5: get $\mathrm{J}$ according to (18).

\section{EXPERIMENT RESULTS}

In this section, we compare our results with that of Tarel 10], He [12], Zhu [14]. These experiments are executed on computer with Inter(R) Core(TM) i5-4460 CPU @3.2GHz Average Gradient (AG) and Structure Similarity Index Metric (SSIM) and processing time. An image with a high AG has a better clarity. A high SSIM represents a higher structure fidelity.

where t0 is set to be 0.1 in case $t$ is too small and then 

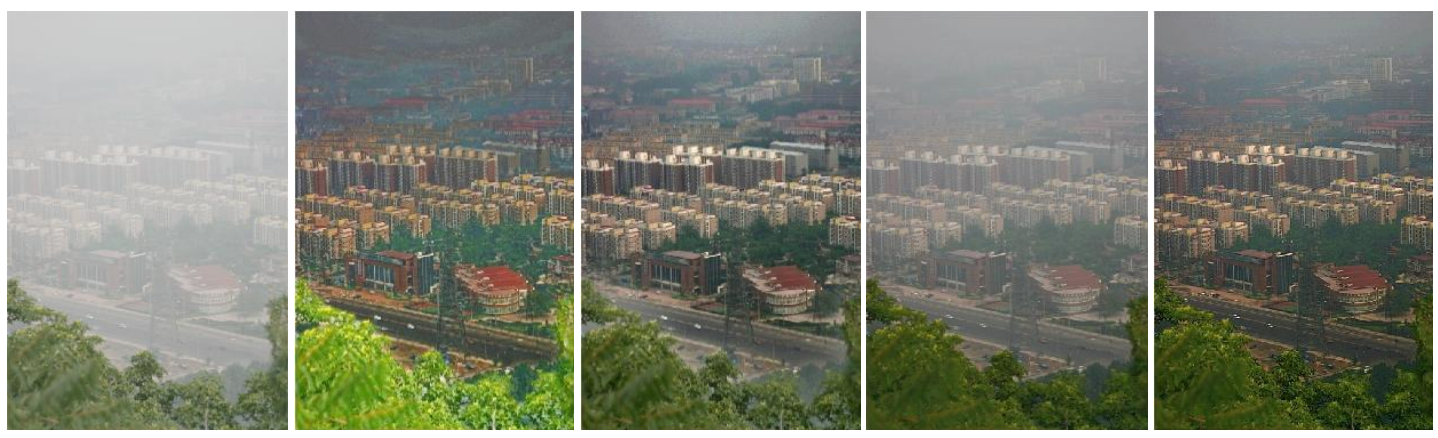

Figure 3. From left to right: input hazy image, restored image by Tarel, He, Zhu, our method.
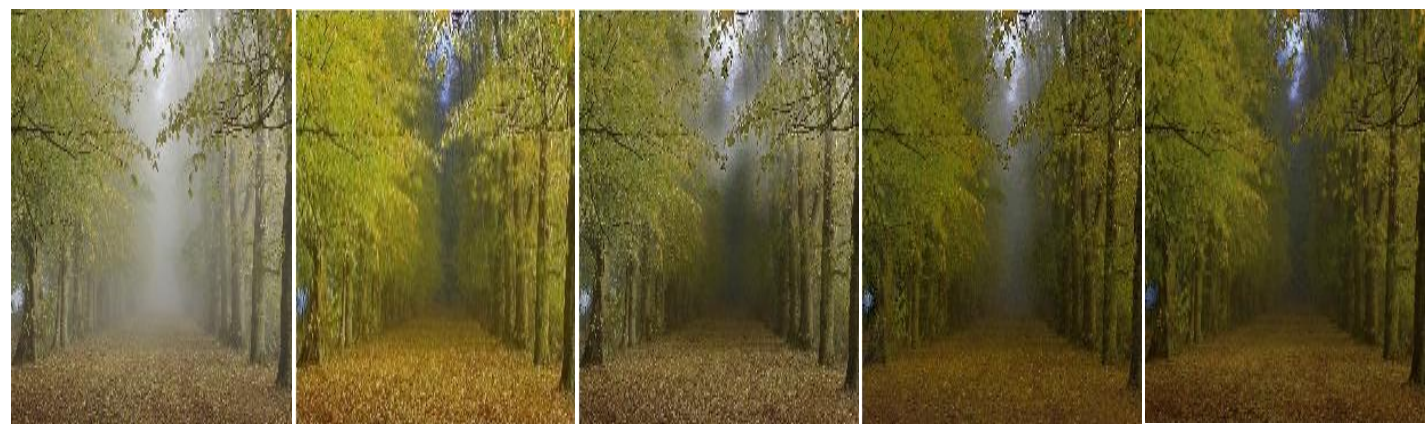

Figure 4. From left to right: input hazy image, restored image by Tarel, He, Zhu, our method.
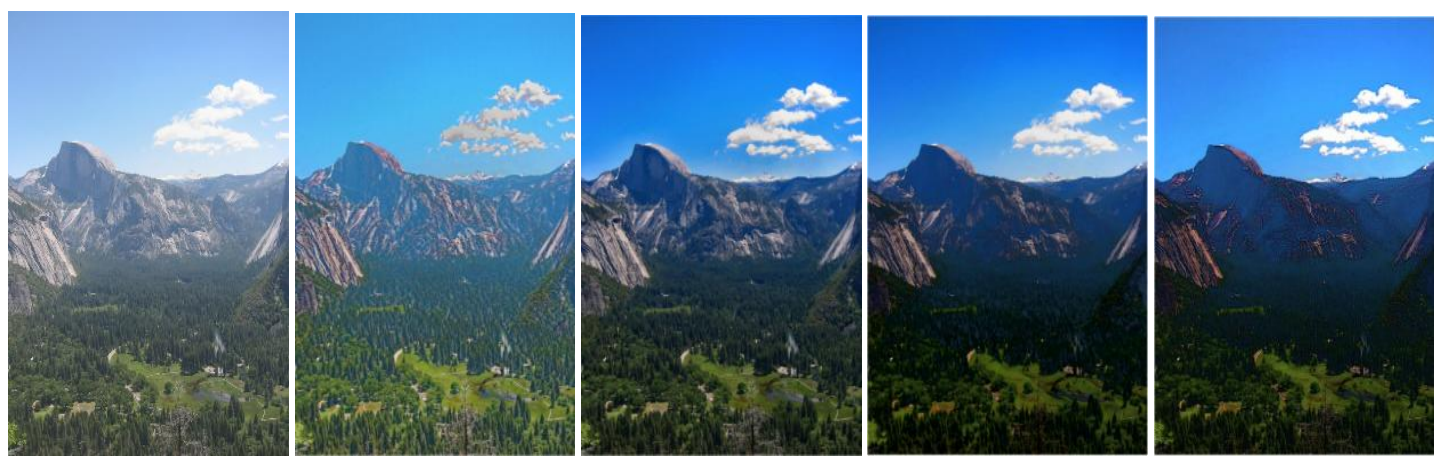

Figure 5. From left to right: input hazy image, restored image by Tarel, He, Zhu, our method.

From Figure 1 to 5, the restored images by Tarel look brighter than others due to a brightness enhancement procedure since the results by Tarel normally look very dim if without brightness enhancement. Results by Tarel and He tend to have some residual haze near edges. Results by Zhu don't show vivid ditail like others which is obvious from Figure 1 and 2 and is demonstrated in Table 1. Furthermore, Zhu's method can't handle dense hazy images well as shown in Figure 3 and 4. Results by our method don't show residual haze and has more vivid detail which is demonstrated in Table 1. From Table 2, we can see that the structure fidelity by our method is comparable to others, which is close to that of Zhu and slightly smaller than that of Tarel and He. The good SSIM value by Zhu for Figure 3 is because it doesn't remove haze sufficiently From Table 3, we can see that the efficiency of our method is comparable to that of Zhu and much better than that of Tarel and He.
TABLE I. AG $/ 10^{-4}$

\begin{tabular}{|c|ccccc|}
\hline Figure & 1 & 2 & 3 & 4 & 5 \\
\hline $\begin{array}{c}\text { Input } \\
\text { image }\end{array}$ & 1.3705 & 0.98419 & 0.44288 & 0.76624 & 0.62302 \\
\hline Tarel & 2.0760 & 1.6064 & 1.2676 & 0.98495 & 0.99939 \\
\hline He & 1.5906 & 1.8013 & 1.0722 & 0.81601 & 0.79585 \\
\hline Zhu & 1.0845 & 1.1276 & 0.55357 & 0.7989 & 0.60714 \\
\hline $\begin{array}{c}\text { Our } \\
\text { method }\end{array}$ & 2.0761 & 2.0298 & 1.2088 & 1.2168 & 1.1606 \\
\hline & & & & & \\
\hline
\end{tabular}


TABLE II. SSIM

\begin{tabular}{c|ccccc}
\hline Figure & 1 & 2 & 3 & 4 & 5 \\
\hline Tarel & 0.9940 & 0.9963 & 0.9877 & 0.9971 & 0.9984 \\
\hline He & 0.9970 & 0.9976 & 0.9887 & 0.9961 & 0.9931 \\
\hline Zhu & 0.9920 & 0.9865 & 0.9934 & 0.9922 & 0.9899 \\
\hline $\begin{array}{c}\text { Our } \\
\text { method }\end{array}$ & 0.9922 & 0.9873 & 0.9848 & 0.9913 & 0.9910 \\
\hline
\end{tabular}

TABLE III.PROCESSING TIME /SECONDS

\begin{tabular}{c|c|c|c|c|c}
\hline Figure & 1 & 2 & 3 & 4 & 5 \\
\hline $\begin{array}{c}\text { Input } \\
\text { image }\end{array}$ & $450 * 440$ & $326 * 1000$ & $600 * 400$ & $768 * 1024$ & $768 * 576$ \\
\hline Tarel & 0.6472 & 1.1161 & 0.7263 & 2.6254 & 1.3868 \\
\hline
\end{tabular}

\section{CONCLUSION}

In this paper, we proposed a new dehazing method using a credibility term based on the saturation channel of HSI color space. We first estimated A using the intensity channel, then removed some high frequency components from W0 and used it as a rough estimation of $t$. Then, we refined the rough estimation by multiplying a credibility term and a white-objects preserving term. We then obtained haze-removed images with more vivid detail and comparable structure fidelity while using very little processing time.

\section{ACKNOWLEDGEMENT}

This research was financially supported by National Nonprofit Industry Research (Funded by the Ministry of land and resources:201411019).

\section{REFERENCES}

[1] S.G. Narasimhan, S.K. Nayar.Vision and the Atmosphere [J]. International Journal of Computer Vision, 2002, 48(3): 233-254.

[2] S.G. Narasimhan, S.K. Nayar.Interactive Deweathering of an Image
Using Physical Models [C]. Proc. IEEE Workshop Color \& Photometric Methods in Computer Vision, 2003.

[3] J. Kopf, B. Neubert, B. Chen, M. Cohen, D. Cohen-Or, O. Deussen,M. Uyttendaele, and D. Lischinski, Deep Photo: Model-based photograph enhancement and viewing $[\mathrm{J}]$. Acm Transactions on Graphics, 2008, 27(5):32-39.

[4] S.G. Narasimhan, S.K. Nayar.Chromatic Framework for Vision in Bad Weather [C]. IEEE Conference on Computer Vision \& Pattern Recongnition, 2000, 1:598-605.

[5] S.G. Narasimhan, S.K. Nayar.Vision in Bad Weather [C]. Proceedings of the 7th IEEE International Conference on Computer Vision, 1999, 2:820-827.

[6] S.G. Narasimhan, S.K. Nayar.Contrast Restoration of Weather Degraded Images [J]. IEEE Transactions on Pattern Analysis and Machine Intelligence, 2003, 25(6):713-724

[7] Y.Y. Schechner, S.G. Narasimhan, and S.K. Nayar. Instant Dehazing of Images Using Polarization [C]. IEEE Computer Society Conference on Computer Vision and Pattern Recognition, 2001, $1: 325-332$

[8] S. Shwartz, E. Namer, and Y.Y. Schechner. Blind Haze Separation [C]. Proc. IEEE Conf. Computer Vision and Pattern Recognition, 2006, 2:1984-1991.

[9] R. Fattal. Single Image Dehazing [J]. ACM Transactions on Graphics, 2008, 27(3):1-9.

[10] J.P. Tarel,N. Hautiere. Fast Visibility Restoration from a Single Color or Gray Level Image [C]. Proceedings of the 12th IEEE International Conference on Computer Vision. Kyoto, Japan, 2009:2201-2208.

[11] Kaiming He, Jian Sun, and Xiaoou Tang. Single Image Haze Removal Using Dark Channel Prior [C]. Proc of the IEEE Computer Society Conference on Computer Vision and Pattern Recognition, 2009:1956-1963.

[12] Kaiming He, Jian Sun, and Xiaoou Tang. Guided Image Filtering [J]. IEEE Transactions on Pattern Analysis and Machine Intelligence, 2013, 35(6):1397-1409.

[13] Z. Wang, Y. Feng. Fast Single Haze Enhancement [J]. Computers and Electrical Engineering, 2014, 40(3):785-795.

[14] Q Zhu, J Mai, and L Shao. A Fast Single Image Haze Removal Algorithm Using Color Attenuation Prior [J]. IEEE Transactions on Image Processing, 2015, 24(11):3522-3533.

[15] V Senthamilarasu,A Baskaran,K Kutty. A New Approach for Removing Haze from Images [C]. Proceedings of the International Conference on Image Processing, Computer Vision, and Pattern Recognition (IPCV), 2014:1-7. 